remission rate of 0.71 after $\mathrm{AED}$ withdrawal, predictors of relapse were adolescent age at onset, symptomatic epilepsies, and an abnormal interictal EEG (Berg AT, Shinnar S. Relapse following discontinuation of antiepileptic drugs: a meta-analysis. Neurology 1994;44:601-608). An identical remission rate was reported by Camfield and colleagues in a study in which patients with absence and minor motor seizures were excluded and seizure type was not of predictive value.(see Progress in Pediatric Neurology II, PNB Publ, 1994, for further discussion of outcome studies in childhood epilepsies). Greater attention to EEG characteristics, especially form of spike-wave complexes and duration of paroxysms, at the time of diagnosis and later at AED withdrawal could be more revealing in future outcome studies.

Accidental injuries, especially bicycle accidents, pose a $27 \%$ risk during absence seizures in children, according to a study of 59 patients at the IWKGrace Health Centre and Dalhousie University, Halifax, Nova Scotia (Wirrell EC, Camfield PR, Camfield CS, Dooley JM, Gordon KE. Accidental injury is a serious risk in children with typical absence epilepsy. Arch Neurol 1996;53:929-932).

\title{
NOCTURNAL FRONTAL LOBE EPILEPSY
}

The electroclinical pattern of 33 patients with familial, autosomal dominant, nocturnal frontal lobe epilepsy was studied, including videopolysomnographic monitoring in 12, at the University of Milano, School of Medicine, Italy. The syndrome is characterized by clusters of brief nocturnal motor seizures during sleep, beginning in childhood and persisting throughout adult life. The motor seizures during sleep varied from thrashing hyperkinetic activity to tonic extension with clonic movements. The most frequently repeated patterns included pelvic thrusting, facial grimacing and moaning, and dystonic posturing. Some had sudden elevation of the head and an expression of fear. Misdiagnoses included benign nocturnal parasomnias, including nightmares, night terrors, and somnambulism. Diurnal episodes in $58 \%$ included generalized shivering followed by loss of consciousness, and complaints of tingling and daytime sleepiness. Interictal and ictal EEGs showed nonspecific patterns (atypical K-complexes), or epileptiform abnormalities (in $58 \%$ of patients), consisting of bilateral or right frontal spikes, during stage 2 non-REM sleep. Normal EEGs were recorded during wakefulness. Both nocturnal and diurnal attacks were controlled by carbamazepine or clonazepam. (Oldani A, Zucconi M, Ferini-Strambi L, Bizzozero D, Smirne S. Autosomal dominant nocturnal frontal lobe epilepsy: electroclinical picture. Epilepsia October 1996;37:964-976). (Reprints: Dr A Oldani, Sleep Disorders Center, IRCCS H San Raffaele, via Prinetti 29, 20127 Milano, Italy).

COMMENT. Nocturnal frontal lobe epilepsy is often misdiagnosed as nightmares, night terrors, or somnambulism. Nocturnal paroxysmal dystonia is also considered in the differential diagnosis. EEGs are frequently nonspecific, and video-polysomnographic monitoring is often essential. If the diagnosis is suspected but unconfirmed by EEG, a trial of antiepileptic drugs may still be warranted.

\section{RISK OF SEIZURE RECURRENCE AFTER FIRST SEIZURE}

The long-term recurrence risk after a first unprovoked seizure was determined in a prospective study of 407 children, followed for a mean of 6.3 years, at the Montefiore Medical Center, Albert Einstein College of Medicine, Bronx, New York. Seizures recurred in 42\%; the cumulative risk of seizure recurrence at $1,2,5$, and 8 year follow-up was $29 \%, 37 \%, 42 \%$, and $44 \%$, 
respectively. One-half recurrences had occurred within 6 months, and almost $90 \%$ within 2 years. Risk factors for seizure recurrences included a remote symptomatic etiology, abnormal EEG, nocturnal seizures, prior febrile seizures, and Todd's paresis. Children with the most favorable prognosis $(21 \%$ recurrence risk after 5 years) had a cryptogenic first seizure while awake and a normal EEG. (Shinnar S, Berg AT, Moshe SL et al. The risk of seizure recurrence after a first unprovoked afebrile seizure in childhood: an extended follow-up. Pediatrics Aug 1996;98:216-225). (Reprints: Shlomo Shinnar MD, Epilepsy Management Center, Montefiore Medical Center, 111 E 210th St, Bronx, NY 10467).

COMMENT. This study is important because of the large number of patients included and the long duration of follow-up. In addition to acquired symptomatic etiology and EEG dysrhythmia, factors frequently associated with an increased risk of seizure recurrence in other studies, sleep related seizures, prior febrile seizures, and Todd's paresis were demonstrated as risk factors. Cryptogenic seizures while awake and a normal EEG were predictive of a good prognosis and a low rate of recurrence.

In a further report, these authors correctly point out that the control of seizures by AEDs and a favorable long-term remission is only part of the goal of therapy. Heightened self-esteem, improved school achievement, and social acceptance by peers are other important considerations. Although the prognosis of epilepsy is determined largely by the underlying cause and associated neurologic abnormalities, the control of seizures by AEDs improves the quality of life and is justification for rational therapy. (Shinnar S, Berg AT. Does antiepileptic drug therapy prevent the development of "chronic" epilepsy? Epilepsia Aug 1996;37:701-708).

\section{SUDDEN UNEXPLAINED DEATH AND EPILEPSY}

The incidence of sudden unexplained death (SUD) among patients less than 50 years old with refractory epilepsy was determined at Boston University Medical Center, Lexington, MA, and Department of Epidemiology, Glaxo Wellcome, Research Triangle Park, NC. Subjects receiving two or more anticonvulsants concurrently were identified from the General Practice Research Database in the UK and their clinical records were reviewed. Of 4150 patients with refractory epilepsy, 612 (15\%) were under 20 years of age. For all subjects, including both idiopathic and acquired epilepsies, the incidence rate of highly probable SUD was $1.5 / 1000$ person-years; for highly probable and possible categories combined, the incidence rate was 2.2/1000 person-years. The respective rates for idiopathic and acquired epilepsy separately were 2.4 and 1.0/1000 person-years. Of a total of 15 subjects with SUD and epilepsy, 8 male and 7 female, 2 (13\%) were younger than 20 years. (Derby LE, Tennis P, Jick H. Sudden unexplained death among subjects with refractory epilepsy. Epilepsia Oct 1996;37:931-935). (Reprints: Dr LE Derby, Boston Collaborative Drug Surveillance Program, 11 Muzzey Street, Lexington, MA 02173).

COMMENT. Idiopathic refractory epilepsy was associated with an estimated risk of sudden unexplained death of 2.4/1000 person-years, whereas the risk with acquired refractory epilepsy was $1.0 / 1000$. A previous study has estimated a higher risk of SUD in patients with acquired epilepsy. The authors concede that relevant information on the cause of epilepsy may have been omitted in some records, leading to an overascertainment of idiopathic cases.

Male sex, the need for multiple AEDs, and use of psychotropic drugs were risk factors for SUD in a Canadian study which showed an overall rate of 